\title{
BioRock: new experiments and hardware to investigate microbe-mineral interactions in space
}

\author{
Claire-Marie Loudon', Natasha Nicholson', Kai Finster², Natalie Leys ${ }^{3}$, Bo Byloos ${ }^{3,4}$, \\ Rob Van Houdt ${ }^{3}$, Petra Rettberg ${ }^{5}$, Ralf Moeller ${ }^{5}$, Felix M. Fuchs ${ }^{5}$, René Demets ${ }^{6}$, \\ Jutta Krause ${ }^{6}$, Marco Vukich ${ }^{7}$, Alessandro Mariani ${ }^{7}$ and Charles Cockell ${ }^{1}$ \\ ${ }^{1}$ UK Centre for Astrobiology, School of Physics and Astronomy, University of Edinburgh, Edinburgh, UK \\ ${ }^{2}$ Department of Bioscience - Microbiology, Ny Munkegade 116, Building 1540, 129, 8000 Aarhus C, Denmark \\ ${ }^{3}$ Microbiology Unit, Belgian Nuclear Research Centre, SCK•CEN, Mol, Belgium \\ ${ }^{4}$ Center for Microbial Ecology and Technology (CMET), Ghent University, Gent, Belgium \\ ${ }^{5}$ Radiation Biology Department, German Aerospace Center (DLR), Institute of Aerospace Medicine, Linder Hoehe, Köln, \\ Germany \\ ${ }^{6}$ ESTEC, Keplerlaan 1, 2201 AZ Noordwijk, Netherlands \\ ${ }^{7}$ Kayser Italia S.r.l., Via di Popogna, 501,57128 Livorno, Italy e-mail: c.s.cockell@ed.ac.uk
}

\begin{abstract}
In this paper, we describe the development of an International Space Station experiment, BioRock. The purpose of this experiment is to investigate biofilm formation and microbe-mineral interactions in space. The latter research has application in areas as diverse as regolith amelioration and extraterrestrial mining. We describe the design of a prototype biomining reactor for use in space experimentation and investigations on in situ Resource Use and we describe the results of pre-flight tests.

Received 20 March 2017, accepted 16 June 2017, first published online 24 July 2017
\end{abstract}

Key words: biofilm, biomining, International Space Station, Mars gravity, microgravity, minerals.

\section{Introduction}

Just as microorganisms can provide useful services on Earth, they can also mediate many processes at the heart of challenges present in the operations of orbital platforms or human outposts on other planetary surfaces (Horneck et al. 2010; Moissl-Eichinger et al. 2016). For example, microbes could be used to perform or aid a wide range of complex tasks such as the extraction of useful minerals from extraterrestrial rocks, generate food and oxygen in a biological life support system, recycle waste and promote plant growth by facilitating soil formation from planetary regolith (Cockell 2010).

Although materials could be taken to other planetary surfaces to establish an outpost, a better way in the long-term is in situ Resource Utilization (ISRU), which uses local resources to establish a presence (Meyer \& McKay 1989; Olsson-Francis \& Cockell 2010; Montague et al. 2012), thus reducing the mass, energy and cost burden and creating local self-sufficiency. Mining minerals is one activity that could be used to derive useful materials from extraterrestrial environments. Biomining is one approach to mining in which microbes could be used to carry out bioleaching, the extraction of useful elements from regolith. Bioleaching/biomining reflects the ability of some microbes to be used to extract useful elements ( $\mathrm{Fe}, \mathrm{Mg}$ and $\mathrm{Ca}$ ) from rock. The microorganisms weather the rock, by mechanisms including production of inorganic or organic acids, and leave the desired cations soluble. This process is already used on the Earth to extract metals from primary ores (Rawlings
2005; Rawlings \& Johnson 2006, 2007). Biomining has ecological and economic benefits over many traditional mining techniques (Schippers et al. 2014), such as reducing waste and the requirement for toxic chemicals to extract metals. If biomining could be applied in extraterrestrial settings then there are possibilities that regolith, such as basalts on the surface on Mars, which contain bioessential cations $\left(\mathrm{Ca}^{2+}, \mathrm{Mg}^{2+}\right.$, $\left.\mathrm{Fe}^{2+}, \mathrm{Fe} 3+, \mathrm{K}^{+}, \mathrm{Na}^{+}\right)$and micronutrients $(\mathrm{Cu}, \mathrm{Mo}$ and $\mathrm{Zn})$, or other more metal-rich ores, could provide resources to sustain human settlement or industries (Raafat et al. 2013). Asteroids, which would be subject to low gravity conditions, are also known to contain useful resources, such as iron, nickel and aluminium along with water, which is an essential resource for biomining (Kryzanowski \& Mardon 1990; Sonter 1997; Busch 2004). As yet, we have no understanding of the effects and the impact of altered gravity regimes, such as microgravity, on the biomining process.

Microbial interactions with planetary regolith could also be used to control and prevent regolith moving downslope (mass movement) in enclosed settings (Liu et al. 2008) or be used as the precursor to soil formation (Mautner 2002; Kral et al. 2004; Lytvynenko et al. 2006).

One of the most distinct differences between the terrestrial and space environment is the lowered gravity. In Martian gravity, this will reduce convection in liquid solutions and in microgravity, there will be no convection at all. Mixing is important for most terrestrial microbe-environment interactions such as waste water treatment (Pell \& Worman 2008). Some terrestrial 
biomining processes require the reactors to be constantly stirred (active stirring) (Rawlings \& Johnson 2007). Mixing is important in bacterial cultures to distribute oxygen and nutrients evenly and to remove waste products from the cells. Therefore, one open scientific question is the extent to which microgravity affects microbe-mineral interactions and the efficacy of substrate dissolution.

Related to our general desire to advance knowledge about how microbes interact with diverse surfaces, including those discussed above, is a need to understand how space conditions affect biofilm growth and structure. Biofilms are found in both beneficial applications (such as the growth of biofilms in waste recycling systems) as well as in deleterious situations (such as on the surface of plastics or within the water distribution system of the International Space Station (ISS)) (Roman \& Minton-Summers 1998; Donlan 2002; Naïtali \& Briandet 2013). Microbial biofilms are important for biomining as one of the most commonly used industrial methods for terrestrial biomining, heap leaching reactors, require the mining microorganisms to grow in biofilms (Rawlings \& Johnson 2007). Microbial biofilms have been found to behave quite differently in low gravity and other low-shear environments than is usually expected from them on Earth (see Nickerson et al. 2004 for a review). Differences in biofilm formation in space have been observed in organisms that have been studied from the medical perspective. These differences include increased biomass production in biofilms of Pseudomonas aeruginosa (Kim et al. 2013b), which is a known spacecraft contaminant and an opportunistic human pathogen that can cause serious illness in immunocompromised persons (Davies 2002). The previous observation that biofilms can form completely novel structures under space conditions, such as the colony and canopy structures observed in Kim et al. (2013a), which arise as a probable result of the absence of flow and gravity during space flight, shows that more experiments to study biofilm formation in space are needed. Investigating biofilm formation and growth has uses in understanding how microbes might grow within life support systems, such as the MELiSSA system, which is under development by the European Space Agency (ESA) (Godia et al. 2002; Hendrickx \& Mergeay 2007; Lasseur et al. 2010; Paradiso et al. 2014).

With numerous commercial space enterprises and continued investment from space agencies, the human presence in space is expanding and with it, the interest in the potential role of microorganisms. An experiment to investigate microbe-mineral interactions is BioRock, proposed in response to the 2009 International Life Sciences Research Announcement: Research Opportunities for Flight Experiments in Space Life Sciences on the ISS and accepted by the ESA (ILSRA-2009-0952) for implementation. This experiment investigates the growth of biofilms on a natural lunar and Martian rock analogue (basalt) under microgravity (near weightlessness) and Martian gravity $(0.38 \mathrm{~g})$. These will be compared with $1 \mathrm{~g}$ (Earth gravity level) controls, one simulated on the ISS and the other carried out on the ground.

The ESA BioRock experiment is designed to test two primary hypotheses: (1) Martian gravity and microgravity affect microbial-induced rock alteration, and (2) Martian gravity and microgravity induce alterations in biofilms formed by microbes associated with rocks.

In this paper, our objective is to describe the BioRock experiment and the design of a prototype extraterrestrial biomining reactor $(\mathrm{BMR})$.

\section{Materials and methods}

\section{Bacterial strains to be used in the study}

As the BioRock experiment is to be flown on the ISS, we were required to select microorganisms that fit certain criteria. The organisms selected should be risk group 1 (representing the lowest risk of pathogenicity to the crew) and have a demonstrated ability to survive desiccation and space flight. It was also deemed important by the BioRock science team that the bacteria chosen should not require growth conditions that may present a danger to the crew, such as a high $\mathrm{pH}$ or the inclusion of toxic chemicals in the growth media. We also required the chosen microorganisms to have a demonstrated presence in a rock or mineral-rich environment.

\section{Selecting the substrate}

As one of the main objectives is to provide precursor experiments that may prove useful in the establishment of off-earth biomining, we therefore searched for a material that would be analogous to a potential target for extra-terrestrial mining.

\section{Selecting a fixative}

A fixative was required to prevent further growth after the completion of the BioRock experiment, but before the experimental hardware could be downloaded from the ISS for analysis. We tested two fixatives that have previously been approved for use on the ISS, RNA later and NOTOXhisto. We tested cooling the fixatives to 0.5 and $4^{\circ} \mathrm{C}$ to test for crystal formation, fixatives that passed this stage were then tested for effectiveness at preventing growth. This test involved incubating the bacteria for 3 weeks as recommended by the BioRock protocol before adding the fixative and counting the number of colony-forming units present after a period of 1 and then 2 weeks. The time delays were chosen as they are selected in the BioRock ESR as the optimum time between the experiment end and download from the ISS.

\section{Pre-flight tests}

To prepare for the launch of the BioRock experiment, the science team completed a series of tests requested by Kayser Italia and ESA as part of a science verification test plan. These tests are designed to ensure that the protocol and the materials used are optimized given the constraints of spaceflight procedures and experimental hardware. Here we describe two of these tests: (1) biocompatibility tests, (2) the test studies on the geometry of bioreactors for biomining experiments in space. We focus on these tests as they formed a critical part of the experimental hardware development and provide useful information for other teams considering similar types of experiments. 


\section{Biocompatibility tests}

These tests are required to ensure that none of the key materials to be used in the manufacture of the hardware has a detrimental effect on the growth of any of the three test organisms selected for use in the BioRock experiment.

The experiment did not measure growth rate, but rather involved investigating whether the materials prevented growth compared with a control in which no material was added. Five millilitres of sterile R2A medium was added to each well of six well plates, a small portion of each material (as provided by Kayser Italia) was added in triplicate to a well (total nine tests of each material, three wells for each BioRock organism). Six microlitres of each of the BioRock organisms was added to each well. The bacteria were allowed to grow for 3 weeks after which the wells were visually examined for signs of reduced growth, which would have been determined by reduced turbidity compared with a control. They were investigated to ensure that there were no zones of clear media around the test materials, which would have indicated that they inhibited the growth of the test organism. The materials tested were: Silicone Xiameter RBL 2004-60, INOX A1 ISO $23382 \mathrm{~h} 8 \times 6$, INOX A4 KI STR DRW 036A151 316, SILICONE SL600W, Silicone Generic, PEEK PVX KISPH-DRW-13, Silicone Oring VMQ80, EPDH Oring, PTFE TEFLON, AISI 420 DIN 6799 23, VITON Oring, INOX A2 DIN 433 M14 AISI 304, INOX A4 DIN 912 M2× 26 AISI 316, Silicone SSP M823.

\section{Geometry of the BMRs}

In order to determine to what extent different geometries and spatial relationships between the components of the hardware affected growth of the model organisms, a number of different preliminary BMRs were built and tested. This also allowed investigation into whether the proposed AF-2400 gas permeable membranes were suitable for the experiment, allowing oxygen to diffuse into the system without microbial contamination.

The geometrical dimensions that were altered in the different designs allowed the following parameters to be explored for their effects on growth: volume of the R2A medium, to see how greater and lesser amounts of nutrient solution affected growth; the membrane effective area, to establish whether differences in the area would significantly limit oxygen availability and therefore growth; the distance between the basalt slide and the membrane, also in regards to checking oxygen availability; and the gravity vector, to ensure the system still functioned in a different orientation. There was a reference configuration BMR to provide a baseline (see rows $1 \mathrm{i}$ to $1 \mathrm{iii}$ in Table 1), its dimensions having been selected based on the space available in the Kubik centrifuge, and additional BMRs with altered dimensions to allow the different parameters to be tested (see rows $2 \mathrm{a}-5 \mathrm{a}$ in Table 1). Figure 1 shows the design of the ground-based reference BMR prototype used for these experiments; the variables on the right show which parts of the BMR had their dimensions altered to provide the required changes in the parameters, apart from the gravity vector parameter, which was achieved by turning the entire BMR upside-down.

Controls were also set up to establish how growth in a simpler system would compare; these were also inoculated with $5 \mathrm{ml}$ of nutrient solution, but were grown in simple glass Erlenmeyer flasks. Biological growth was measured through an optical density spectrometer, which detects how much light is passing through the sample. The higher the concentration of bacteria, the less light gets through. At inoculation, each BMR and control started with an optical density of 0.038 . After 3 weeks of growth, the liquid was sluiced around the rock with pipettes to homologise the distribution of the culture, and then extracted for spectroscopy at $600 \mathrm{~nm}$.

\section{Design and layout of the experimental hardware}

The hardware for the BioRock experiment was developed under an ESA contract by Kayser Italia as primary contractor. The specification required the development of a novel hardware that could be used to study microbe-mineral interactions in space. The hardware was required to allow for the two levels of containment required for safety reasons for an organism on the ISS (NASA guidelines for microbial research aboard the ISS, 2014) and was requested to fit inside the KUBIK centrifuge. Designing the hardware was the first attempt to construct miniature prototype BMRs. It was designed with the specifications that the bacteria can be launched to the ISS in a desiccated state and that the experiment will not become active until a liquid medium is injected into the chamber containing the bacteria. This active experimental change can be ended through the injection of a fixative.

\section{Spaceflight strategy}

A detailed in flight protocol for BioRock guided development of the hardware. Of key importance was optimization of the number of samples that could be run at one time and a focus on automation to reduce the crew time devoted to the experiment.

\section{Results and discussion}

Bacterial strains - the rationale for the strains chosen

The microbes studied in the Biorock experiment are Sphingomonas desiccabilis (University of Edinburgh), Bacillus subtilis NCIB 3610 (German Aerospace Center (DLR) Cologne, Germany) and Cupriavidus metallidurans CH34 (SCK-CEN). As this experiment is to be carried out on the ISS we were required to select strains that could test in principle the extraction of minerals from rock in microgravity rather than those that are necessarily the most efficient biominers. Many of the most efficient biomining organisms such as Acidithiobacillus ferrooxidans require an extremely low $\mathrm{pH}$, usually between pH 1-2 (Valdés et al. 2008). We chose not to attempt to recreate such acidic growth conditions on the ISS due to safety concerns. We selected microorganisms that have a demonstrated presence in a rock or mineral-rich environment and an ability to survive the desiccation required for 
Table 1. Effects of geometry on microbial growth in reactors. The different dimensions of the BioMining Reactors and optical densities of microbial growth determined after 3 weeks

\begin{tabular}{|c|c|c|c|c|c|c|c|}
\hline BMR & $\begin{array}{l}\text { Volume of } \\
\text { R2A }(\mu 1)\end{array}$ & $\begin{array}{r}\text { Depth } \\
(\mathrm{mm})\end{array}$ & $\begin{array}{l}\text { Diameter } \\
(\mathrm{mm})\end{array}$ & $\begin{array}{l}\text { Membrane effective } \\
\text { area }\left(\mathrm{mm}^{2}\right)\end{array}$ & Orientation & $\begin{array}{l}\text { Optical } \\
\text { density: } 0 \mathrm{~h}\end{array}$ & $\begin{array}{l}\text { Optical density: } \\
3 \text { weeks }\end{array}$ \\
\hline $\mathrm{C} 1$ & 5000 & 9 & 36 & $\mathrm{n} / \mathrm{a}$ & Up & 0.038 & 0.683 \\
\hline $\mathrm{C} 2$ & 5000 & 9 & 36 & $\mathrm{n} / \mathrm{a}$ & $\mathrm{Up}$ & 0.038 & 0.707 \\
\hline C3 & 5000 & 9 & 36 & $\mathrm{n} / \mathrm{a}$ & Up & 0.038 & 0.549 \\
\hline li & 5000 & 10 & 26.2 & 10 & Up & 0.038 & 0.308 \\
\hline lii & 5000 & 10 & 26.2 & 10 & Up & 0.038 & 0.697 \\
\hline 1iii & 5000 & 10 & 26.2 & 10 & $\mathrm{Up}$ & 0.038 & 0.338 \\
\hline $2 \mathrm{a}$ & 10000 & 10 & 36.4 & 10 & Up & 0.038 & 0.444 \\
\hline $2 b$ & 16000 & 10 & 45.7 & 10 & $\mathrm{Up}$ & 0.038 & 0.554 \\
\hline $3 a$ & 5000 & 10 & 26.2 & 20 & Up & 0.038 & 0.639 \\
\hline $3 b$ & 5000 & 10 & 26.2 & $\mathrm{n} / \mathrm{a}$ & $\mathrm{Up}$ & 0.038 & 0.581 \\
\hline $3 c$ & 5000 & 10 & 26.2 & 5 & $\mathrm{Up}$ & 0.038 & 0.553 \\
\hline $4 a$ & 5000 & 27 & 16 & 10 & Up & 0.038 & 0.804 \\
\hline $4 b$ & 5000 & 4 & 41.5 & 10 & Up & 0.038 & 0.440 \\
\hline $4 c$ & 5000 & 17 & 20.1 & 10 & $\mathrm{Up}$ & 0.038 & 0.751 \\
\hline $5 \mathrm{a}$ & 5000 & 10 & 26.2 & 10 & Down & 0.038 & 0.421 \\
\hline
\end{tabular}

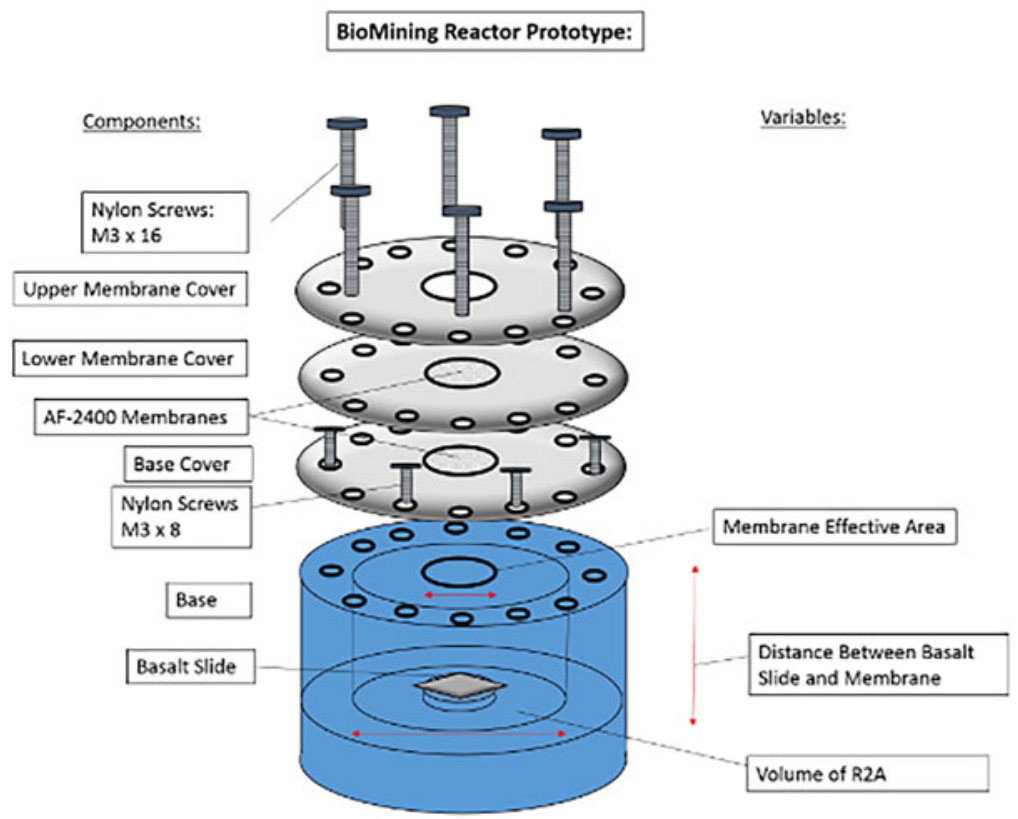

Fig. 1. A prototype laboratory BMR used to investigate the effects of geometry on microbial growth and to test membrane efficacy.

their upload to the ISS. Preliminary results from all the three of the collaborating BioRock groups show evidence of elemental leaching from basalt in the presence of these test organisms (unpublished results). These organisms allow us therefore to test the role, in principle, that altered gravity may play in off-earth mining processes using organisms that are all deemed to be risk group 1 (representing the lowest risk of pathogenicity to the ISS crew) and which can be grown in standard laboratory conditions that do not pose a threat to safety on board the space craft.

S. desiccabilis is a Gram-negative, non-spore-forming bacterium, which was isolated from soil crusts in the Colorado plateau (Reddy \& Garcia Pichel 2007). Sphingomonas species have been shown to increase the leaching rate of $A$. ferrooxidans when added to a joint bacterial community (Huang et al. 2013) and the natural growth of $S$. desiccabilis on desert crust mineral surfaces makes it an ideal organisms to study microbe-mineral interaction As a non-motile bacterium it can provide insights into the role of bacterial motility for biofilm formation in microgravity and Martian gravity when compared with the other two selected motile microbes.

B. subtilis is a Gram-positive, motile, spore-forming bacterium found naturally in soil and vegetation. It is widely studied as both a bacterial model organism and for secreted enzyme production (Krishnappa et al. 2013; Michna et al. 2014; Losick 2015). B. subtilis is particularly noted for its production 
of endospores and its ability to survive harsh environments (Horneck et al. 2010). B. subtilis has also been found to weather granite (Song et al. 2007) in order to extract essential minerals to live. Understanding its behaviour in micro-gravity is of high importance as it has been found previously as a common contaminant on the ISS (Nickerson et al. 2004).

C. metallidurans $\mathrm{CH} 34$ is a Gram-negative, motile, nonspore forming bacterium and a model organism to study metal resistance and tolerance. It is isolated from different metal-contaminated environments (Diels \& Mergeay 1990; Brim et al. 1999; Goris et al. 2001) as well as volcanic basalt (Kelly et al. 2010) and granite rock (Sahl et al. 2008). In addition, $C$. metallidurans strains have been recovered from other anthropogenic environments including medically-relevant sources (Langevin et al. 2011; Van Houdt et al. 2012) and space habitats (Mijnendonckx et al. 2013; Mora et al. 2016). Its interaction with basalt as well as its response to spaceflight conditions has previously been studied (Olsson-Francis et al. 2010; Mergeay \& Van Houdt 2015; Bryce et al. 2016). The organism has also been shown to be able to grow under spaceflight conditions with minimal changes to its proteome and with minor effects on cell physiology (Leys et al. 2009).

\section{Substrate to be studied}

Basalt has been selected as the rock substrate for the experiment (the elemental composition of the exact basalt rock used in the BioRock experiment is given in Table 2). Basalt is an igneous rock, which is highly abundant on Earth as well as on the surface of the Moon (Ruzicka et al. 2001) and Mars (McMahon et al. 2013; McSween 2015) making it a potential target for extraterrestrial biomining. Basalt is mineralrich and the rock surface is usually porous enough to provide a good support for bacterial colonization (McMahon et al. 2013). Rich bacterial communities have been found living in basalt on Earth (Cockell et al. 2009; Templeton et al. 2009; Kelly et al. 2011). Furthermore, interactions between heterotrophic bacteria and basaltic substrates are known to occur and have been studied and measured previously (e.g. $\mathrm{Wu}$ et al. 2007; Bryce et al. 2015). Olivine is the dominant mineral in basalt and an olivine-based biological iron-oxidation has been observed in organisms from a Mars analogue basaltic environment (Popa et al. 2012).

\section{Selecting a fixative}

In the initial BioRock design, RNAlater was selected as the fixative. This was chosen as it has already been approved for use on the ISS. However, a concern was raised about the suitability of this chemical for spaceflight as it had previously been known to form crystals at low temperatures and such cold temperatures are often encountered by space experiment payloads during upload. Crystals would jam the injection mechanisms in the hardware. If this was found to be the case, then RNAlater would not be suitable for this space experiment. We tested the effects of cooling to 4 and $0.5^{\circ} \mathrm{C}$, the lowest temperature that the samples would encounter during upload to the ISS. We found that at $4^{\circ} \mathrm{C}$ the RNAlater did indeed form crystals, but
Table 2. The elemental composition of the BioRock Basalt: The elemental composition of the basalt slides determined by fluorescence measurement upon X-ray irradiation on a Spectro Xepos apparatus

\begin{tabular}{llc}
\hline Symbol & Element & Concentration (\%) \\
\hline $\mathrm{Na}_{2} \mathrm{O}$ & Sodium & 1.92 \\
$\mathrm{MgO}$ & Magnesium & 10.00 \\
$\mathrm{Al}_{2} \mathrm{O}_{3}$ & Aluminium & 15.35 \\
$\mathrm{SiO}_{2}$ & Silicon & 47.48 \\
$\mathrm{P}_{2} \mathrm{O}_{5}$ & Phosphorus & 0.128 \\
$\mathrm{~K}_{2} \mathrm{O}$ & Potassium & 0.162 \\
$\mathrm{CaO}$ & Calcium & 11.69 \\
$\mathrm{TiO}_{2}$ & Titanium & 1.344 \\
$\mathrm{MnO}^{\mathrm{Fe}_{2} \mathrm{O}_{3}}$ & Manganese & 0.186 \\
\hline
\end{tabular}

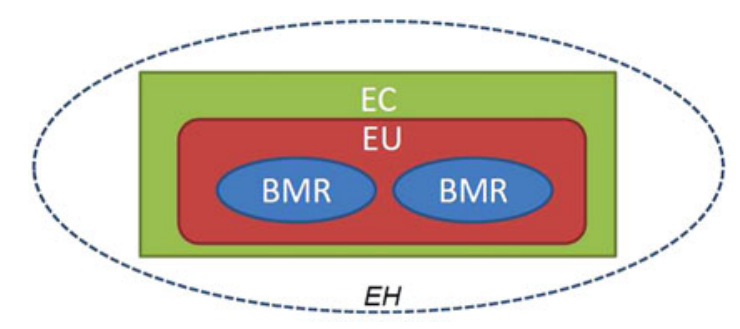

Fig. 2. General schematic of Experimental Hardware (EH). The figure shows a schematic of the experimental hardware for the BioRock experiment. Two biomining reactors (BMRs) are enclosed within an experimental unit (EU), which in turn is enclosed within an experimental container (EC). This creates the two levels of containment.

when returned to room temperature these disappeared within $24 \mathrm{~h}$.

We then tested the alcohol-based fixative NOTOXhisto and found it to be effective in reducing growth, although it could not completely stop growth. NOTOXhisto will be added into the culture chamber after the 3-week experiment is completed. The experimental containers (ECs) will then be stored at $4^{\circ} \mathrm{C}$ to further slow any additional growth until they can be downloaded from orbit. After download the science team will meet the samples at the landing site and will add formaldehyde as a more effective fixative.

\section{Biocompatibility tests}

We found that none of the materials tested had a negative effect on growth for any of the test organisms. It was therefore concluded that all the materials provided to the science team by Kaiser Italia were suitable for use in the BioRock hardware.

\section{Geometry of the BMRS}

Results from the 3-week test are shown in Table 1. From the reference configuration results alone (rows 1i-1iii in Table 1) we can see that there is some variation in how $S$. desiccabilis grows over time, but that every BMR allowed for growth. 

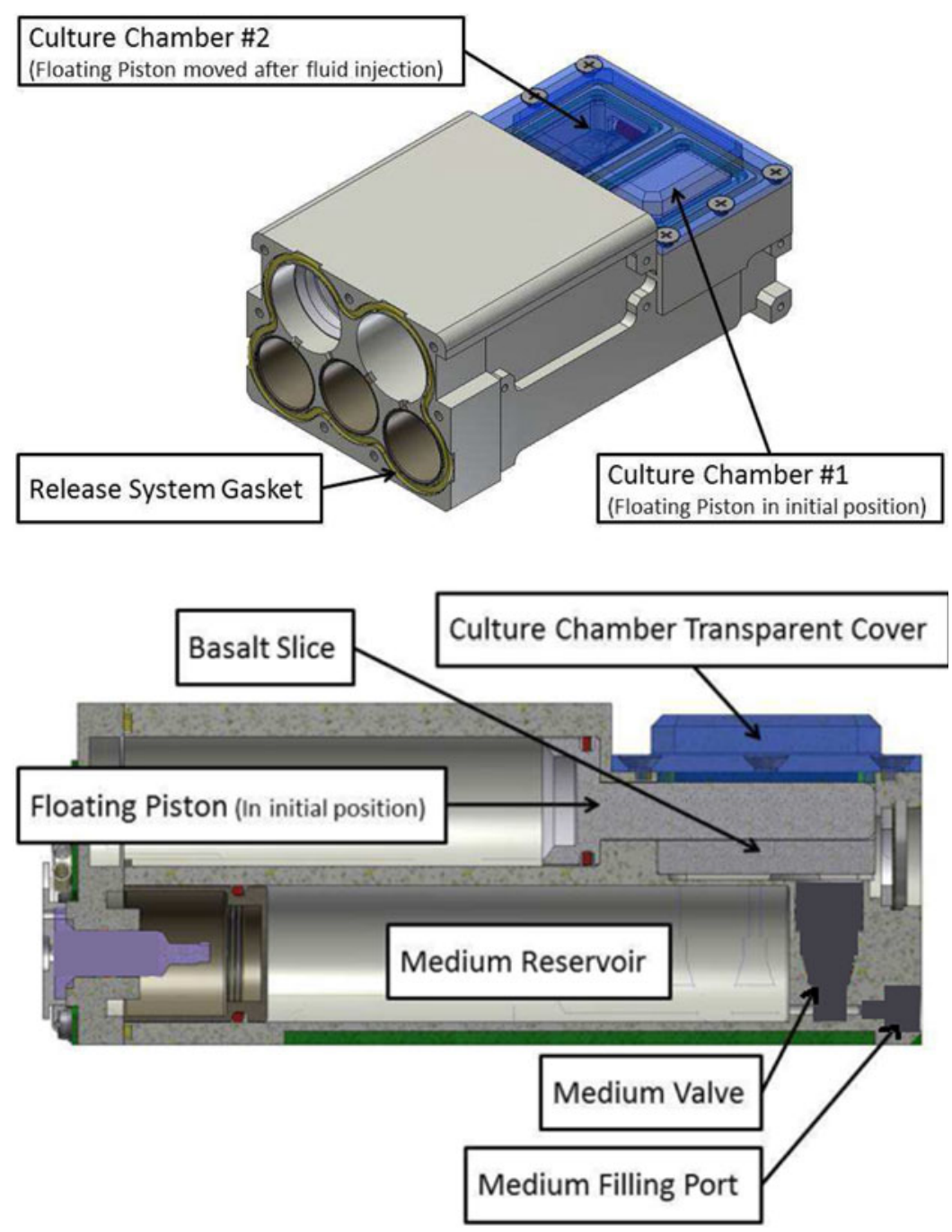

Fig. 3. BioRock experimental hardware system A. The figure shows a detailed image of the first design of the hardware. It shows how the liquids required for the experiment, such as the medium, can be transferred between chambers using pistons.

Growth occurred in all versions of the BMR, indicating that the AF-2400 membranes functioned as expected, and that none of the proposed dimensions were detrimental to the growth of the model organisms.

A one-way ANOVA test was run on the results to establish whether the results indicated a difference between the controls and the BMR treatments, and also to assess whether the different BMR parameters had a significant effect on the growth rates of the organisms.

The statistics were run on R-3.1.2 for Windows. The ANOVA test gave a $P$-value of 0.2637 .

\section{Features of the experimental hardware}

The BioRock experiment has allowed us to develop novel hardware that can generically be used to study microbe-mineral interactions and biofilm formation in space. This hardware consists of 'biomining reactors' composed of three chambers: a culture chamber, a medium reservoir chamber and a fixative reservoir chamber. Two of these BMRs can be contained within each experimental unit (EU). The EU is in turn stored inside an EC creating the two levels of containment required for safety reasons for an organism on the ISS (NASA guidelines for microbial research aboard the ISS, 2014) an illustration of this is shown in Fig. 2. Thirty-six of these BMRs will be prepared for the BioRock experiment and the ECs have been designed to the specification that all 36 reactors can be placed simultaneously on two KUBIK incubators present on the ISS. The movement of liquids between the three chambers of the reactor is achieved with a series of springs and pistons, which are controlled by the astronauts electronically.

In the BioRock design and construction phase, two potential design solutions were obtained. We have chosen solution B for the BioRock experiment, but we present both solutions here for the benefit of other research groups, since either may have benefits for other experiments.

In solution A, the culture chamber with the basalt slide contains the piston, which takes up the headspace (Fig. 3). Upon the start of the experiment, the piston is withdrawn, microbial culture medium is drawn into the culture chamber and the experiment commences. In solution B (Fig. 4), the culture 

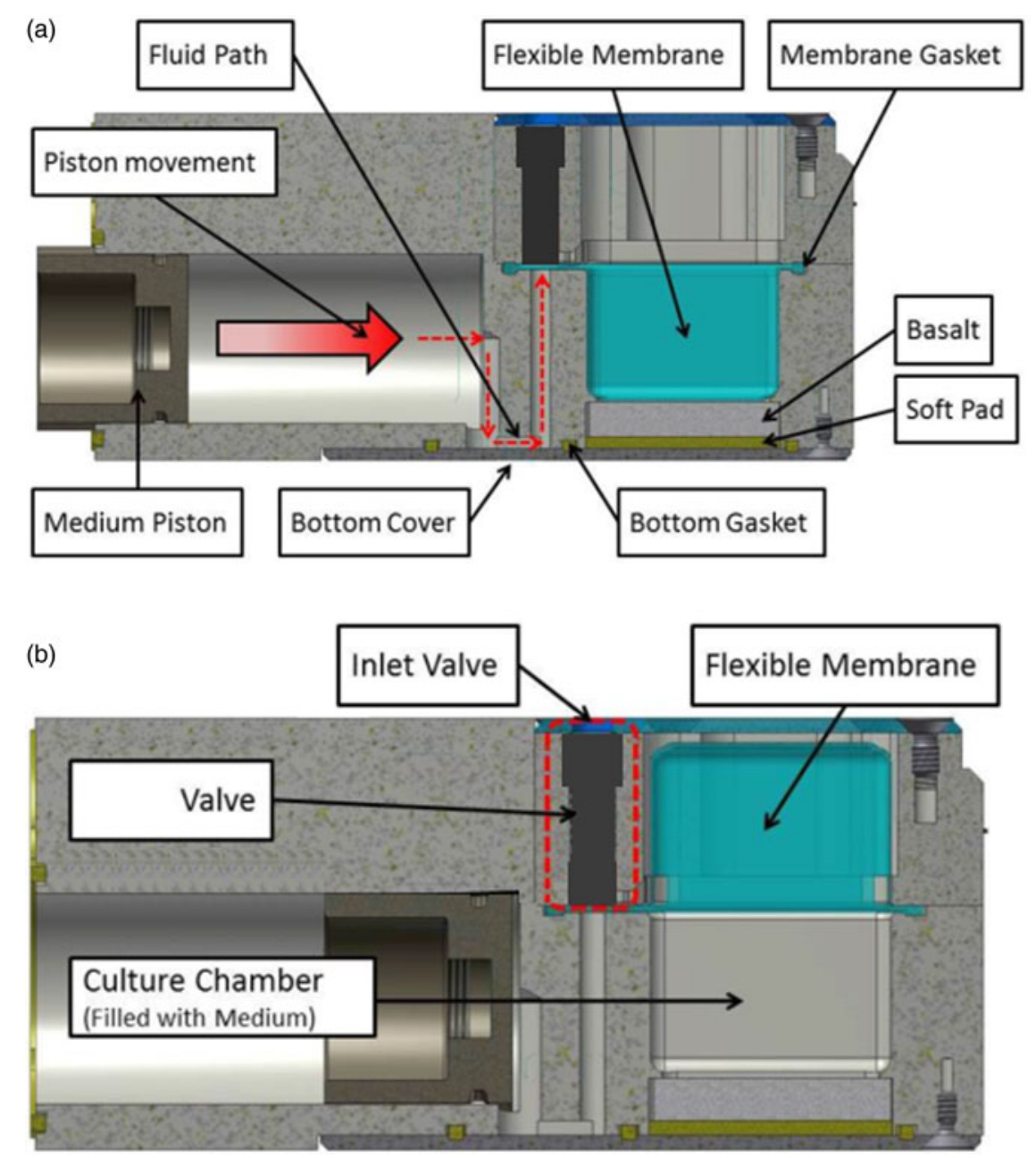

Fig. 4. BioRock experimental hardware system B. (a) Schematic showing BMR before the liquid medium is injected from the medium reservoir into the culture chamber. (b) The hardware after injection of the medium. The flexible membrane flips inside out when liquid is injected into the culture chamber to allow gas exchange to occur just above the bacterial culture. This membrane is also transparent to allow for photographs to be taken of the biofilm on the basalt slice.

container consists of a semi-permeable, deformable membrane (for example, material XIAMETER RBL-2004-60 Silicone) system from which gas has been removed. When fluid is injected, the membrane expands to fill the culture chamber (Figs. 4 and 5). The advantage of solution B is that the amount of gas in the culture chamber can be minimized and the membrane, which is on five sides of the chamber, improves oxygen access to the culture.

\section{Operations before and during flight}

BioRock will use the KUBIK centrifuge system, which is a cube-shaped small $\left(37 \times 37 \times 37 \mathrm{~cm}^{3}\right)$ portable incubator that can provide 1 and $0.38 \mathrm{~g}$ (Martian gravity) on board of the ISS by changing the rotor speed of its centrifuge, (KUBIK factsheet, ESA).

The experiment is prepared in triplicate using the three organisms as depicted in Fig. 6. Each experimental condition (microgravity, Martian gravity $(0.38 \mathrm{~g})$ and $1 \mathrm{~g})$ has three BMRs and a control without organisms to evaluate rock leaching in the absence of microorganisms. A ground-based $1 \mathrm{~g}$ control will also be run in identical hardware in parallel to the space experiment using the same batches of bacteria.

\section{Preparation of materials pre-flight}

S. desiccabilis, B. subtilis spores and C. metallidurans $\mathrm{CH} 34$ are desiccated onto basalt slides, which are then locked in the culture chamber of the BMR. Each basalt slide is $1 \times 1 \mathrm{~cm}^{2}$ in size and $2 \mathrm{~mm}$ thick. Five $\mathrm{ml}$ of sterile R2A medium will be loaded and sealed into each of the medium reservoirs. $\mathrm{R} 2 \mathrm{~A}$ is a growth medium its composition is $\left(\mathrm{g}^{-1}\right)$ : yeast extract 0.5 ; peptone 0.5 ; casamino acids 0.5 ; glucose 0.5 , soluble starch 0.5 , Na-pyruvate $0.3 ; \mathrm{K}_{2} \mathrm{HPO}_{4} 0.3 ; \mathrm{MgSO}_{4} .7 \mathrm{H}_{2} \mathrm{O} 0.05$ at $\mathrm{pH}$ 7.2. One $\mathrm{ml}$ of the fixative NOTOXhisto will be loaded and sealed into the fixative reservoirs. The hardware is then ready for flight and will be delivered to ESA.

\section{During flight-arrangement of the BMRs in the KUBIK}

The general concept of how the BMRs are to be operated during the experiment is shown in Fig. 7. 


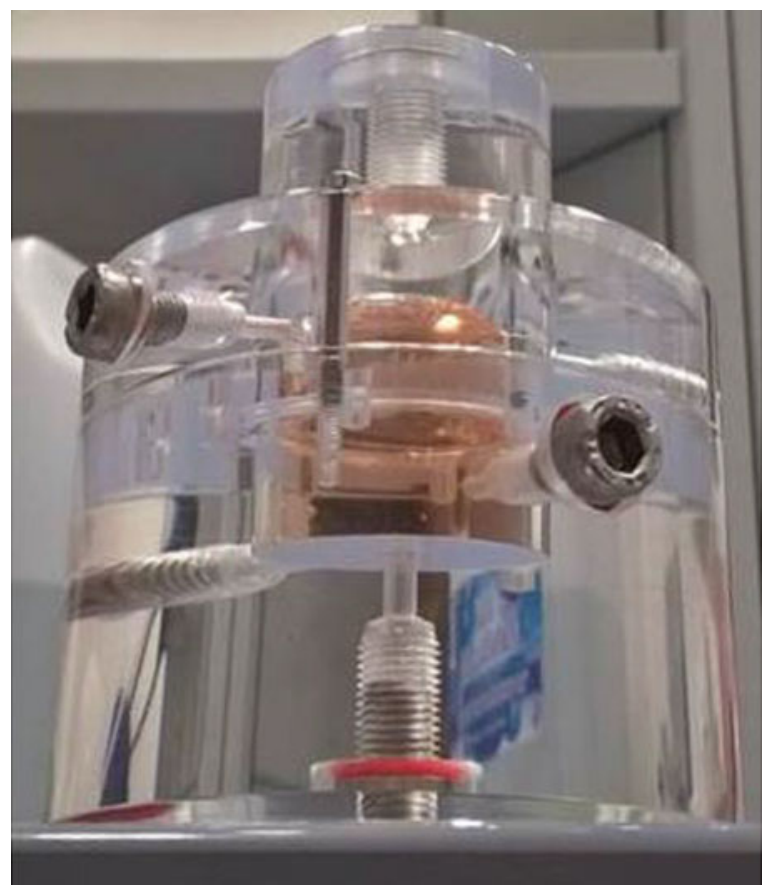

Fig. 5. Membrane testing for system $B$. The testing of the flexible membrane for prototype B being carried out at Kayser Italia. This test demonstrated that very little pressure was required in order to cause the membrane to flip inside out.
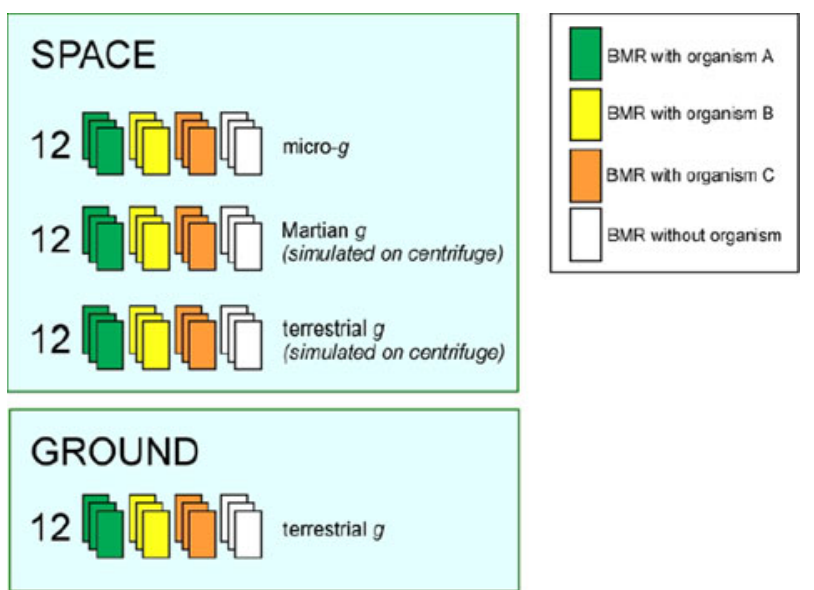

Fig. 6. The experimental setup concept for the BioRock experiment using three organisms.

There are two ways in which the BioRock experiment could be accomplished using the KUBIK centrifuge. One setup is to use one KUBIK, the second possibility is to use two KUBIK centrifuges (both configurations shown in Fig. 8).

We have chosen to use two KUBIK centrifuges because all samples are treated in exactly the same way with respect to storage time and conditions during transport, upload, storage on the ISS before and after the experiment in space, download and transport to the investigators laboratories, but we show both configurations for the potential benefit of other research groups. The configuration designs illustrate how a large experiment can potentially be managed using limited on-orbit resources, particularly in cases where only one piece of experimental hardware is available and yet an experiment cannot be fitted in, in its entirety.

Figure 9 shows a diagram of the BioRock ECs as they will be arranged for the experiment within the KUBIK centrifuges. The BioRock experiment will use two KUBIK machines to create two altered gravity levels. One centrifuge will be spun to create a gravity level of $1 \mathrm{~g}$ similar as a terrestrial g-level control; a second centrifuge will be spun to create a level of gravity of $0.38 \mathrm{~g}$ similar to that of Mars. Finally, BMRs will also be placed in holders within the KUBIK chamber but outside the centrifuge in order to study the effects of microgravity.

\section{Initiation of the experiment}

The active phase of the experiment will be started by activating the hardware using electrical controls and injecting medium from the medium reservoir chamber into the culture chamber to revive the desiccated bacteria. At this stage, the astronauts will remove the BMRs from the KUBIK centrifuges and they will photograph the reactors before returning the BMRs to the KUBIK and leaving the experiment to run for 3 weeks at approximately $20^{\circ} \mathrm{C}$. After the incubation period is completed, the astronauts stop the experiment using the electrical control, which will trigger the injection of the fixative into the culture chamber to prevent further growth. This mechanism is shown in detail in Fig. 10. The astronauts will then complete a second photo session of the BMRs before storing them at $4^{\circ} \mathrm{C}$ for their return to Earth. These photo sessions are intended to reassure the ground researchers that the experiment went to plan before the ground analyses begin. The first photo session will show if the experiment was initiated as expected (i.e. the culture media was successfully injected into culture chamber), whilst the second photo shoot will allow the researchers to verify if the biofilms were growing on the rock in space in case the cells grow in an unexpected location or if the cells become dislodged during download from the ISS.

\section{Importance of BioRock}

BioRock will provide important precursor experiments for the establishment of extraterrestrial biomining. One of the particular strengths of this experiment is that it studies three microorganisms with a link to rock weathering using the exact same protocol, hardware and media. This makes the results comparable. This is important, as different variables in separate experiments have been blamed for discrepancies found between previous studies of microbes and biofilms in space (Nickerson et al. 2004; Kim et al. 2013b), even if the conditions are suboptimal for some of the selected microorganisms. Knowing if these microbes behave differently under altered gravity conditions or if there are patterns such as an increase or decrease in mineral extraction from rock in altered gravity present in all three would have implications for our confidence in using microorganisms as components of life support systems or as potential biominers.

One example of these problems is that some researchers have reported increased cell density of Escherichia coli in microgravity whilst others have not (Gasset et al 1994; Brown et al. 2002). 


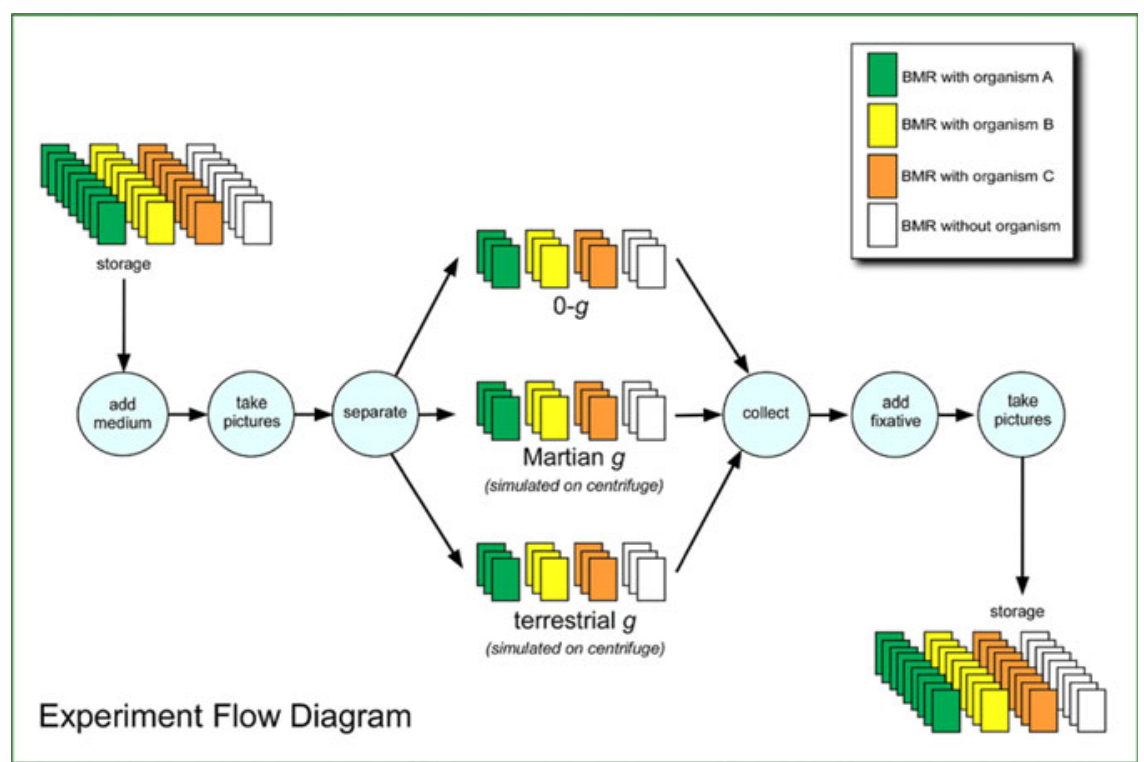

Fig. 7. The protocol concept for the BioRock experiment.

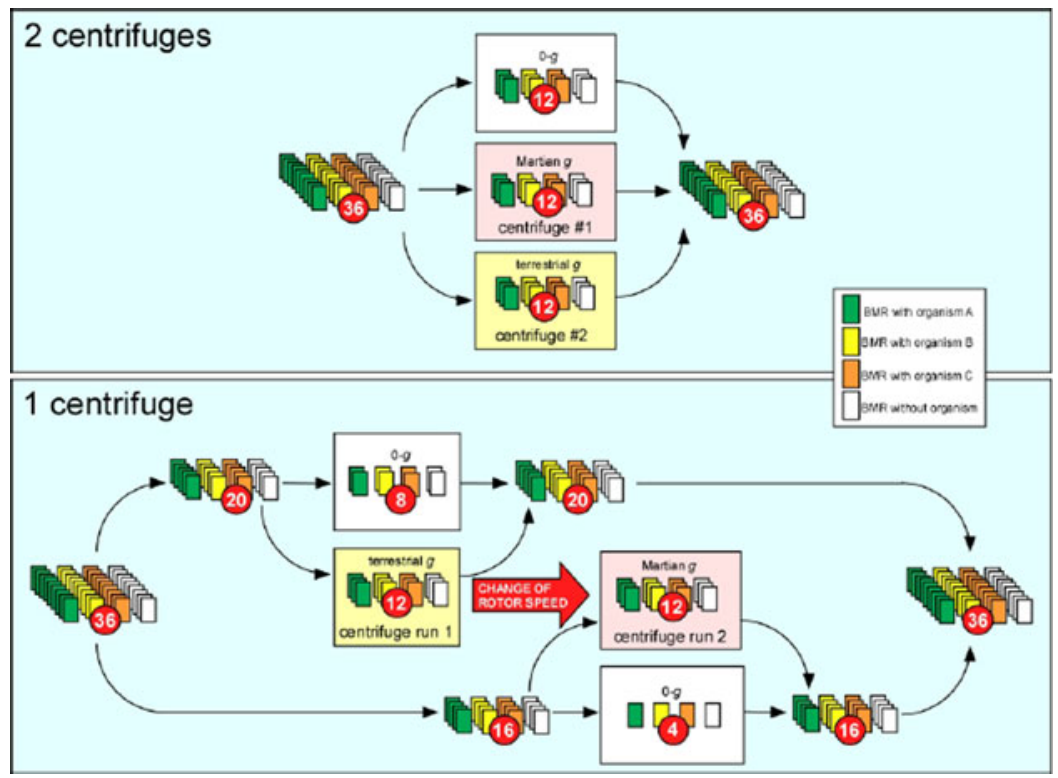

Fig. 8. Two possible ways of running the BioRock experiment using one or two KUBIK centrifuges.

Kim et al. (2013b) predict that these differences are due to changes in the growth medium and culture conditions. The same authors also postulated that different bacterial species may respond to the microgravity environment differently and by testing three unrelated organisms, the BioRock experiment is also able to offer some insight for this hypothesis.

\section{Future work - post-flight tests}

The samples will be processed as soon as possible after their return to Earth by members of the BioRock science team. The biofilms will be further fixed with formaldehyde to ensure no further growth occurs between their download to Earth and their investigation in the laboratory. The following post-flight analyses are planned:

Biofilm structure: The effects of microgravity and Martian gravity on the biofilm structure will be studied using scanning electron microscopy (Hawser et al. 1998), confocal microscopy (De Beer et al. 1997), Raman spectroscopy (Millo et al. 2011) and staining techniques. The structures of the biofilms will be compared between the organisms from the flight experiment and the ground controls, a comparison will also be carried of the biofilms formed by the different test organisms.

Elemental release rates: The extent of bioleaching under each of the conditions will be studied through analyses of the release rates of all major cations and trace elements into solution. These solutions will be examined by inductively coupled 
KUBIK \#01

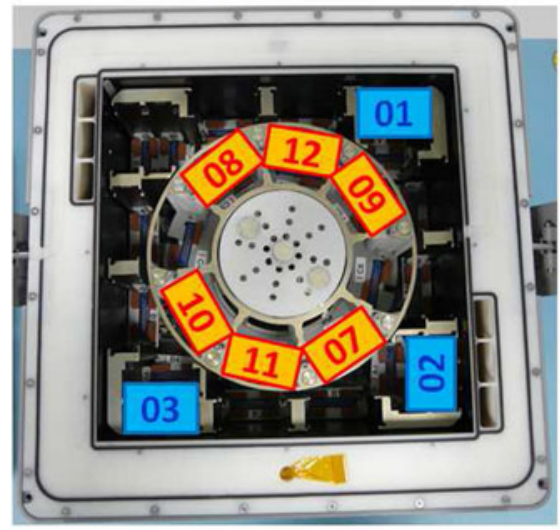

KUBIK\#02

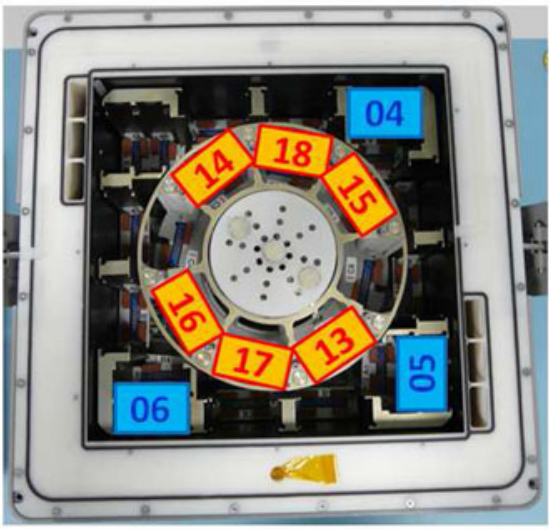

Fig. 9. The BioRock setup within the KUBIK centrifuges. The figure shows how the two KUBIK units will be arranged during the BioRock experiment. The numbered boxes show the experimental containers (ECs) that are described above and shown in Fig. 2. As they are not being spun in the centrifuge, the ECs numbered between 1 and 6 will be exposed to the microgravity conditions of the ISS. The ECs numbered between 7 and 12 are in one of the centrifuges and this will be spun to $0.38 \mathrm{~g}$, which will create gravity conditions similar to those found on Mars. The ECs labelled from 13 to 18 are in a separate centrifuge and will be kept in $1 \mathrm{~g}$ gravity conditions like those present on Earth.

(a)

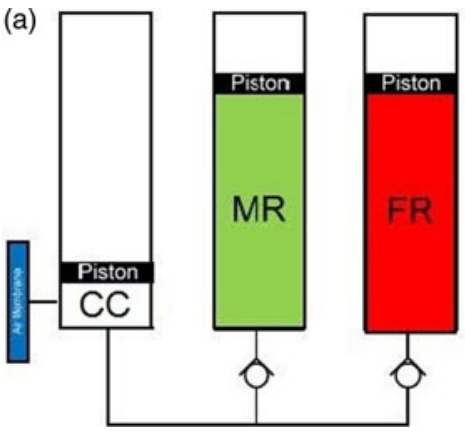

(b)

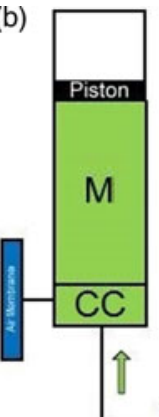

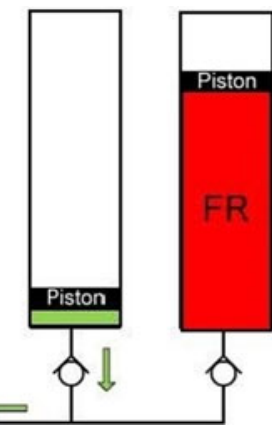

(c)

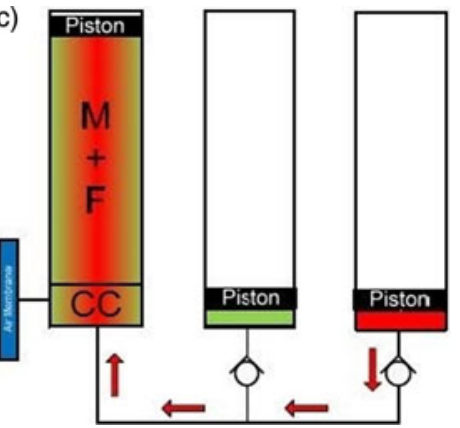

Fig. 10. Schematic representation for the internal workings of the BioRock experimental hardware. In stage (a), the three chambers within the bioreactor are as they would be at launch to the ISS. In the CC (Culture chamber) there will be a uniformly cut slice of basalt rock with one of the test microorganisms desiccated upon it. The medium in the media reservoir (MR) and the fixative in the fixative reservoir (FR) are kept separate. In stage (b) the media is injected from its reservoir into the culture chamber. This will revive the desiccated bacteria and begin the 3-week experimental period. Stage (c) reflects the end of the experiment when the fixative is injected from its reservoir into the culture chamber and prevents any further growth of the bacteria. This is how the bioreactor will be stored until down load from the ISS.

plasma mass and optical emission spectrometry (ICP- MS/ OES), (Maezato et al. 2012). This result will aid the determination of if and how microgravity alters the ability to extract minerals from the rock for the investigated organisms. If differences can be observed in the elemental release rates during flight as compared with the ground control then any microorganisms that are effective in terrestrial biomining would need to be tested in micro- or lowered gravity before they could be deemed suitable for use in extraterrestrial biomining.

\section{Conclusions}

The BioRock experiment designed for the ISS offers the opportunity to study biomining and biofilm formation in three different microorganisms in parallel under altered gravity conditions. The findings will provide conclusions for the possibility of obtaining minerals using biomining on other planetary surfaces such as the Moon and Mars and on the growth of organisms under altered gravity regimes generally. The experiment requires the development of miniature BMRs, which can be used to test microbial growth, microbe-mineral interactions, other experiments focused on ISRU and microbial behaviour in the space environment.

\section{Acknowledgements}

C. M. L. and C. S. C. were supported by the STFC grant number: ST/M001261/1, N. N. is a doctoral student at the University of Edinburgh supported by a studentship from the UK space agency. P. R., R. M. and F. M. F. were supported by the DLR grant FuE-Projekt 'ISS LIFE' (Programm RF-FuW, Teilprogramm 475). F. M. F. is a doctoral student of the Helmholtz Space Life Sciences 
Research School (SpaceLife), German Aerospace Center (DLR) Cologne, Germany, which was funded by the Helmholtz Association (Helmholtz-Gemeinschaft) over a period of 6 years (Grant No. VH-KO-300) and received additional funds from the DLR, including the Aerospace Executive Board and the Institute of Aerospace Medicine. The authors would like to thank two anonymous reviewers whose comments greatly improved this manuscript.

\section{References}

Brim, H., Heyndrickx, M., de Vos, P., Wilmotte, A., Springael, D., Schlege, H.G. \& Mergeay, M. (1999). Syst. Appl. Microbiol. 22(2), 258-268.

Brown, R.B., Klaus, D. \& Todd, P. (2002). Microgravity Sci. Technol. 13, 24-29. Bryce, C.C., Le Bihan, T., Martin, S.F., Harrison, J.P., Bush, T., Spears, B., Moore, A., Leys, N., Byloos, B. \& Cockell, C.S. (2016). Environ. Microbiol. 18(4), 1110-1121.

Busch, M. (2004). J. Brit. Interplanet. Soc. 57, 301-305.

Cockell, C.S. (2010). Trends Microbiol. 18, 308-314.

Cockell, C.S., Olsson, K., Knowles, F., Kelly, L., Herrera, A., Thorsteinsson, T. \& Marteinsson, V. (2009). Geomicrobiol. J. 26, 491-507.

Davies, J.C. (2002). Paediatr. Respir. Rev. 3(2), 128-134.

De Beer, D., Stoodley, P. \& Lewandowski, Z. (1997). Biotechnol. Bioeng. 53, 151-158.

Diels, L. \& Mergeay, M. (1990). Appl. Environ. Microbiol. 56(5), 1485-1491. Donlan, R.M. (2002). Emerging Infectious Dis. 8, 881-890.

Gasset, G., Tixador, R., Eche, B., Lapchine, L., Moatti, N., Toorop, P. \& Woldringh, C. (1994). Res. Microbiol. 145, 111-120.

Godia, F., Albiol, J., Montesinois, J.L., Pérez, J., Creus, N., Cabello, F., Mengual, X., Montras, A. \& Lasseur, C.H. (2002). J. Biotechnol. 99, 319-330.

Goris, J., De Vos, P., Coenye, T., Hoste, B., Janssens, D., Brim, H., Diels, L., Mergeay, M., Kersters, K. \& Vandamme, P. (2001). Int. J. Syst. Evol. Microbiol. 51, 1773-1782.

Hawser, S.P., Baillie, G.S. \& Douglas, L.J. (1998). J. Med. Microbiol. 47, 253-256.

Hendrickx, L. \& Mergeay, M. (2007). Curr. Opin. Microbiol. 10, 231-237.

Horneck, G., Klaus, D.M. \& Mancinelli, R.L. (2010). Microbiol. Mol. Biol. Rev. 74, 121-156.

Huang, T., Gong, W.Q., Bao, G.M. \& Lei, S.M. (2013). Adv. Mater. Res. 823, 613-617.

Kelly, L. et al. (2011). Microb. Ecol. 62, 69-79.

Kelly, L.C., Cockell, C.S., Piceno, Y.M., Andersen, G.L., Thorsteinsson, T. \& Marteinsson, V. (2010). Microb. Ecol. 60(4), 740-752.

Kim, W. et al. (2013a). PLoS ONE 8, 4.

Kim, W. et al. (2013b). BMC Microbiol. 13, 241

Kral, T.A., Bekkum, C.R. \& McKay, C.P. (2004). Orig. Life Evol. Biosph. 34, 615-626.

Krishnappa, L., Dreisbach, A., Otto, A., Goosens, V.J., Cranenburgh, R.M., Harwood, C.R., Becher, D. \& van Diji, J.M. (2013). J. Proteome Res. 12, 4101-4110.

Kryzanowski, T. \& Mardon, A. (1990). Can. Mining J. 111, 43.

Langevin, S., Vincelette, J., Bekal, S. \& Gaudreau, C. (2011). J. Clin. Microbiol. 49(2), 744-745.

Lasseur, C.H., Brunet, J., de Weever, H., Dixon, M., Dussap, G., Godia, F., Leys, N., Mergeay, M. \& Van Der Straeten, D. (2010). Gravit. Space Biol. 23, 3-12.

Leys, N., Baatout, S., Rosier, C., Dams, A., s'Heeren, C., Wattiez, R. \& Mergeay, M. (2009). Antonie Van Leeuwenhoek 96, 227-245.

Liu, Y.D., Cockell, C.S., Wang, G., Hu, C.X., Chen, L. \& De Philippis, R. (2008). Astrobiology 8, 75-86.

Losick, R. (2015). J. Biol. Chem. 290(5), 2529-2538.

Lytvynenko, T. et al. (2006). Res. Microbiol. 157, 87-92.
Maezato, Y., Johnson, T., McCarthy, S., Dana, K. \& Blum, P. (2012). J. Bacteriol. 194(24), 6856-6863.

Mautner, M.N. (2002). Icarus 158, 72-86.

McMahon, S., Parnell, J., Ponicka, J., Hole, M. \& Boyce, A. (2013). Astron. Geophys. 54, 1.17-1.21.

McSween, H.Y. (2015). Am. Miner. 100, 2380-2395.

Mergeay, M. \& Van Houdt, R. (eds) (2015). Springerbriefs in Biometals. Springer International Publishing, Switzerland.

Meyer, T.R. \& McKay, C.P. (1989). J. Brit. Interplan. Soc. 42, 147-160.

Michna, R.H., Commichau, F.M., Tödter, D., Zschiedrich, C.P. \& Stülke, J. (2014). Nucleic Acids Res. 42(Database issue), D692-D698.

Mijnendonckx, K., Provoost, A., Ott, C.M., Venkateswaran, K., Mahillon, J., Leys, N. \& Van Houdt, R. (2013). Microb. Ecol. 65(2), 347-360.

Millo, D., Harnisch, F., Patil, S.A., Ly, H.K., Schröder, U. \& Hildebrandt, P. (2011). Angew. Chem. Int. Ed. 50, 2625-2627.

Moissl-Eichinger, C., Cockell, C.S. \& Rettberg, R. (2016). FEMS Microbiol. Rev. 40, 722-737.

Montague, M., McArthur, G.H., Cockell, C.S., Held, J., Marshall, W., Sherman, L.A., Wang, N., Nicholson, W.L., Tarjan, D.R. \& Cumbers, J. (2012). Astrobiology 12, 1135-1142.

Mora, M., Perras, A., Alekhova, T.A., Wink, L., Krause, R., Aleksandrova, A., Novozhilova, T. \& Moissl-Eichinger, C. (2016). Microbiome 4(1), 65. doi: 10.1186/s40168-016-0217-7.

Naïtali, M. \& Briandet, R. (2013). Biofilm - La société des microbes. Biofutur 341, 23-33.

Nickerson, C.A., Ott, C.M., Wilson, J.W., Ramamurthy, R. \& Pierson, D.L. (2004). Microbiol. Mol. Biol. Rev. 68, 345-361.

Olsson-Francis, K. \& Cockell, C.S. (2010). Planet. Space Sci. 58, 12791285.

Olsson-Francis, K., Van Houdt, R., Mergeay, M., Leys, N. \& Cockell, C.S. (2010). Geobiology 8(5), 446-456.

Paradiso, R., De Micco, V., Buonomo, R., Aronne, G., Barbieri, G. \& De Pascale, S. (2014). Plant Biol. 16, 69-78.

Pell, M. \& Worman, A. (2008). Encyclopedia Ecol. 16, 426-444.

Popa, R., Smith, A.R., Popa, R., Boone, J. \& Fisk, M. (2012). Astrobiology 12, 9-18.

Raafat, K., Burnett, J., Chapman, T. \& Cockell, C.S. (2013). Astron. Geophys. 54, 5.10-5.12.

Rawlings, D.E. (2005). Microbial Cell Factories 4, 1-15.

Rawlings, D.E. \& Johnson, B. (2006). Biomining. Springer, Heidelberg.

Rawlings, D.E. \& Johnson, B. (2007). Microbiology 153, 315-324.

Reddy, G.S.N. \& Garcia- Pichel, F. (2007). Int. J. Syst. Evol. Microbiol. 57, $1028-1034$.

Roman, M.C. \& Minton- Summers, S. (1998). Life Support Biosph. Sci. 5(1), $45-51$.

Ruzicka, A., Snyder, G.A. \& Taylor, L.A. (2001). Geochim. Cosmochim. Acta 65, 979-997.

Sahl, J.W. et al. (2008). Appl. Environ. Microbiol. 74(1), 143-152.

Schippers, A., Hedrich, S., Vasters, J., Drobe, M., Sand, W. \& Willscher, S. (2014). In Geobiotechnology I Metal-related Issues: Biomining: Metal recovery from ores with microorganisms, ed. Schippers, S., Glombitza, F. \& Sand, W., pp. 1-47. Springer, Heidelberg.

Song, W., Ogawab, N., Oguchic, C.T., Hattad, T. \& Matsukuraa, Y. (2007). CATENA 70(3), 275-281.

Sonter, M.J. (1997). Acta Astronaut. 41, 637-647.

Templeton, A.S., Knowles, E.J., Eldridge, D.L., Arey, B.W., Dohnalkova, A.C., Webb, S.M., Bailey, B.E., Tebo, B.M. \& Staudigel, H. (2009). Nat. Geosci. 2, 872-876.

Valdés, J., Pedroso, I., Quatrini, R., Dodson, R.J., Tettelin, H., Blake, R., Eisen, J.A. \& Holmes, D.S. (2008). BMC Genomics 9, 597. doi: 10.1186/ 1471-2164-9-597.

Van Houdt, R., Monsieurs, P., Mijnendonckx, K., Provoost, A., Janssen, A., Mergeay, M. \& Leys, N. (2012). BMC Genomics 13, 111.

Wu, L.L., Jacobson, A.D., Chen, H.C. \& Hausner, M. (2007). Geochim. Cosmochim. Acta 71, 2224-2239. 